\title{
Transvaginal Endoscopic Appendectomy
}

\author{
Eung Jin Shin, Gui Ae Jeong, Jun Chul Jung, Gyu Seok Cho, Chul Wan Lim, Hyung Chul Kim, \\ Ok Pyung Song \\ Department of Surgery, Soonchunhyang University Bucheon Hospital, Soonchunhyang University College of Medicine, Bucheon, Korea
}

Since Kalloo and colleagues first reported the feasibility and safety of a peroral transgastric approach in the porcine model in 2004, various groups have reported more complex natural orifice transluminal endoscopic surgery (NOTES) procedures, such as the cholecystectomy, splenectomy and liver biopsy, in the porcine model. Natural orifice access to the abdominal cavity, such as transgastric, transvesical, transcolonic, and transvaginal, has been described. Although a novel, minimally invasive approach to the abdominal cavity is a peroral endoscopic transgastric approach, there are still some challenging issues, such as the risk of infection and leakage, and the method of gastric closure. Hybrid-NOTES is an ideal first step in humans. Human hybrid transvaginal access has been used for years by many surgeons for diagnostic and therapeutic purposes. Here, we report a transvaginal flexible endoscopic appendectomy, with a 5-mm umbilical port using ultrasonic scissors in a 74-year-old woman with acute appendicitis.

\section{Keywords: Endoscopy; Appendectomy; Hybrid NOTES}

\section{INTRODUCTION}

Since Kalloo et al. [1] introduced the transgastric abdominal approach using endoscopy in animal experiments in 2004, natural orifice transluminal endoscopic surgery (NOTES) has been attempted worldwide and has improved tremendously in a short time. NOTES which was reported only in animal experiments, started to be applied to humans in 2007. The Strasburg group [2] performed the hybrid method of a transvaginal endoscopic cholecystectomy using a $5-\mathrm{mm}$ abdominal trocar successfully for the first time, and Rao et al. [3] in India did a video presentation of a transgastric appendectomy through the stomach. In Korea, Shin et al. [4] did a video presentation of a hybrid transvaginal endoscopic appendectomy using a 5$\mathrm{mm}$ abdominal trocar in 2007 for the first time. Afterward, Kim et al. [5] reported a transgastric abdominal inspection by using

Received: August 26, 2010 Accepted: September 30, 2010

Correspondence to: Eung Jin Shin, M.D.

Department of Surgery, Soonchunhyang University Bucheon Hospital, 1174

Jung-dong, Wonmi-gu, Bucheon 420-767, Korea

Tel: +82-32-621-5249, Fax: +82-32-621-5018

E-mail:colon@schmc.ac.kr

(C) 2010 The Korean Society of Coloproctology

This is an open-access article distributed under the terms of the Creative Commons Attribution NonCommercial License (http://creativecommons.org/licenses/by-nc/3.0) which permits unrestricted noncommercial use, distribution, and reproduction in any medium, provided the original work is properly cited. the NOTES technique in an animal experiment, and Lee et al. performed a NOTES appendectomy in gynecological patients by using only endoscopic instruments [6].

The ultimate goal of NOTES is to perform abdominal surgery, such as a cholecystectomy and an appendectomy, transorally through the stomach wall. If a complete NOTES could be performed, theoretically, since abdominal wounds would be absent, pain should be less than it would be for conventional surgical methods, esthetic effects would be superior, and patients could, thus, return to work eariler. Nevertheless, NOTES has numerous limitations, such as a longer operation time due to technical difficulties and a shortage of proper equipment. In addition, in regard to closure of the perforation site within the natural orifice, an acceptable closure method is not yet present; hence, the development of proper closure method should be pursued.

Currently, the posterior vaginal wall is available as a safe route, Numerous surgeries, such as a gynecological transvaginal hysterectomy, have already been performed by using a transvaginal approach, and perforation areas could be safely closed with manual suturing. This is a report of a hybrid endoscopic appendectomy that was combined with a transvaginal approach using colonoscopy and an abdominal approach with a $5-\mathrm{mm}$ working port. This study was approved by the ethics committee of Soonchunhyang University (SCHBC-IRB-07-24), and informed consent was obtained from the patient. 


\section{CASE REPORT}

A 74-year-old female patient was admitted via the outpatient clinic for abdominal pain in the right lower quadrant area that had persisted for two days. The patient had no specific medical illnesses and family history. Based on history taking and physical examination, acute appendicitis was suspected; thus, laboratory tests and abdominal ultrasonography were performed. On the blood test, leucocytes were increased to $12,000 / \mathrm{mm}^{3}$, and other test results were within normal ranges. On the chest and the abdominal radiographs, there were no specific findings. On abdominal sonography, the appendix was thickened to 10 $\mathrm{mm}$, thus confirming acute appendicitis. Before surgery, the potential risks of the transvaginal approach, such as vaginal hemorrhage, infection, and dyspareunia, were explained sufficiently to the patient, and informed consents were taken after their approval.

One hour prior to surgery, a second generation antibiotic was administered intravenously. Under general anesthesia, the pa-

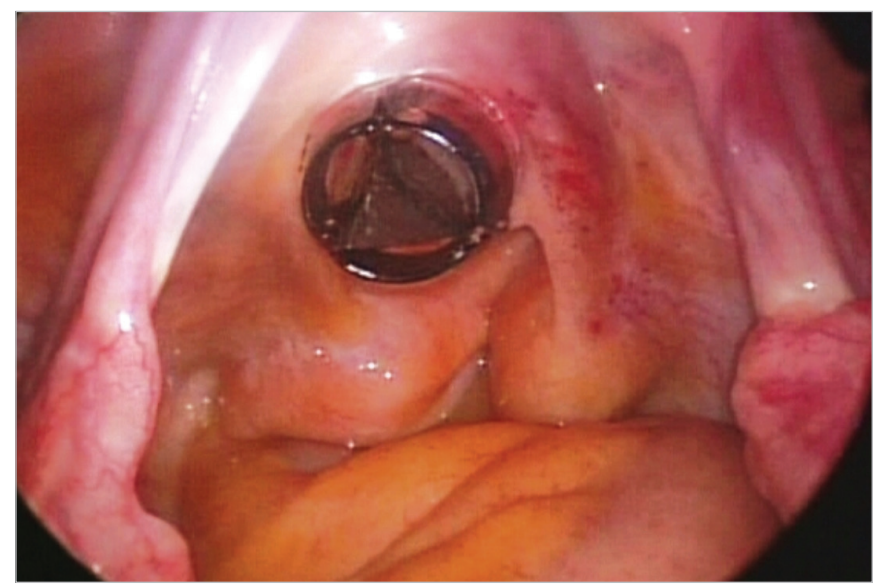

Fig. 1. Transvaginal trocar introduced through the culdotomy site.

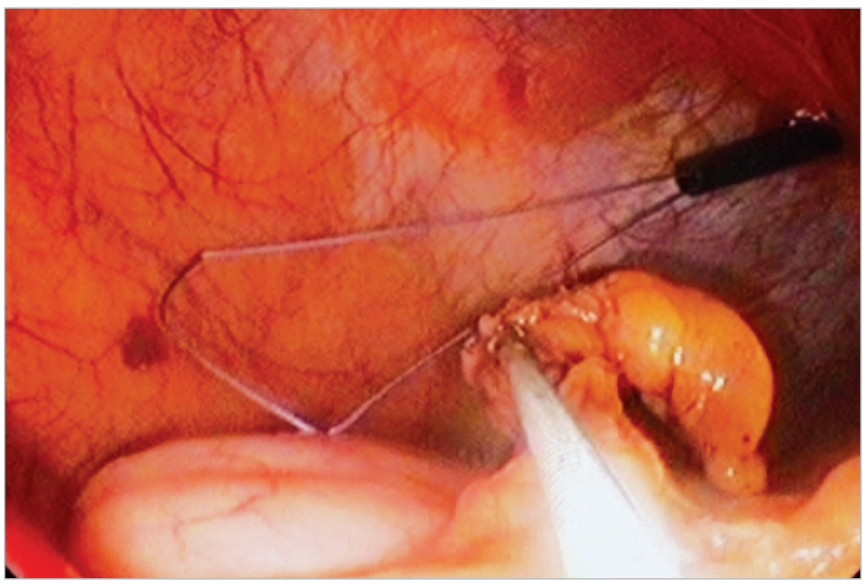

Fig. 3. Laparoscopic ligature inserted through the umbilical trocar. tient was placed in the lithotomy position. A transverse 5-mm skin incision was made along the inferior margin of the umbilicus, and a 5-mm trocar was inserted. Under $\mathrm{CO}_{2}$-pneumoperitoneum, a laparoscopic camera was inserted (Fig. 1). The surgeon moved to the patient's perineal side (between legs) and made a $15-\mathrm{mm}$ incision in the posterior vaginal wall and inserted a 15-mm trocar under laparoscopic vision. Through the 15-mm trocar, a colonoscope was inserted. The monitor view was switched from the laparoscopic camera to the colonoscopic view, and the peritoneal cavity was assessed. After laparoscopic camera in the 5-mm abdominal trocar had been removed, an ultrasonic scalpel was inserted.

The inflamed appendix in the right lower abdomen was examined using the colonoscope, and using endoscopic graspers, we dissected the appendix from the adjacent bowels by lifting the tip of the appendix (Fig. 2). The mesoappendix was dissected using the ultrasonic scalpels by coagulation modes, and hemo-

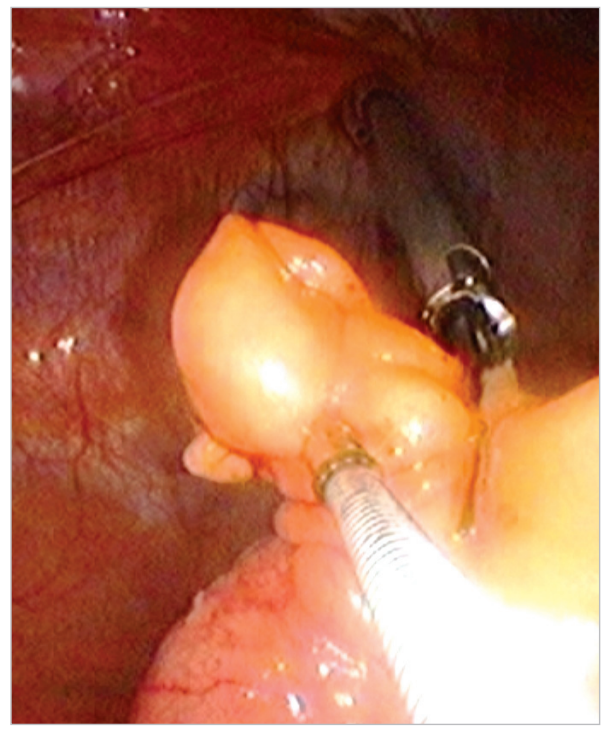

Fig. 2. Appendix grasped by using endoscopic forceps and divided from the mesoappendix by using ultrasonic scissors.

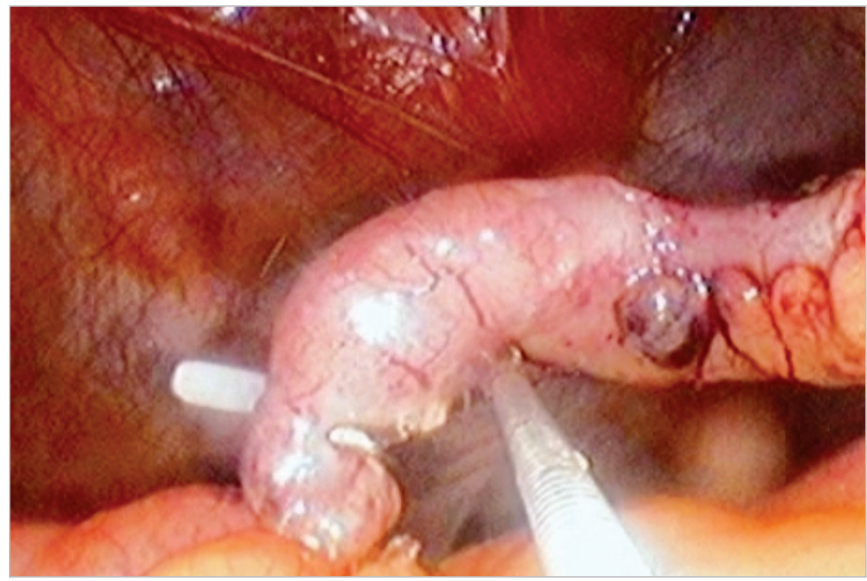

Fig. 4. Appendix divided by using ultrasonic scissors. 


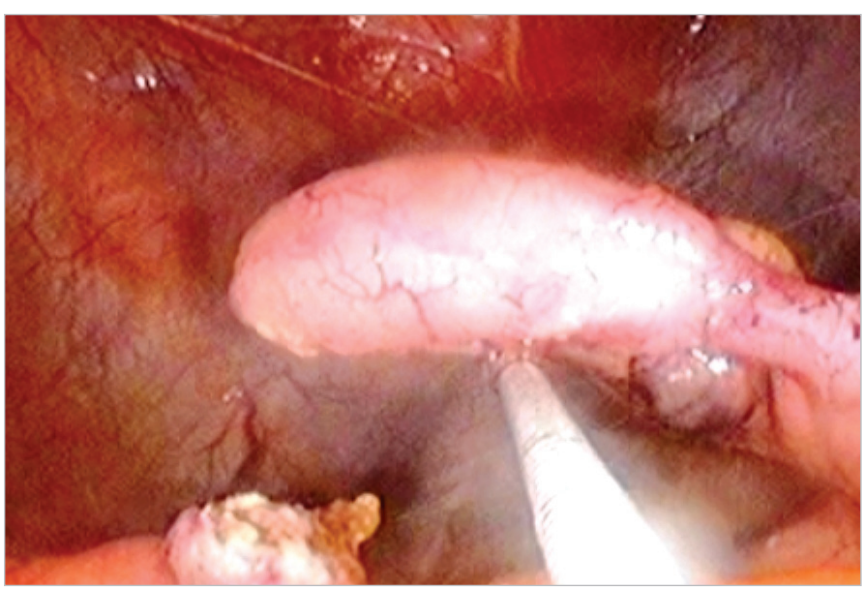

Fig. 5. Extraction of the appendix through the transvaginal trocar.

stasis of the appendiceal artery was confirmed. The base of the appendix was ligated using laparoscopic loop ligatures (Fig. 3). The ligated appendix was transsected with the ultrasonic scalpels (Fig. 4) and moved to outside the abdomen, together with the colonoscope, through the tranvaginal trocar by using endoscopic graspers (Fig. 5). After hemostasis had been confirmed, the perforation site in the posterior vaginal wall was sutured manually. The operation time was 60 minutes.

The patient started to eat from one day after surgery and was discharged 3 days after surgery without wound infection, fever, pelvic pain, urinary difficulty or other specific complication. The vaginal wound dressing was done at 1 day after surgery, similar to the wound care after obstetrical gynecological surgery, and antibiotic suppositories were inserted for one week. At the ambulatory follow-up observation performed two weeks after discharge, the abdominal and vaginal wound had healed well, and the patient had no specific complaints.

\section{DISCUSSION}

Together with robotic surgeries, NOTES is considered to be the next-generation minimally-invasive surgery; thus, numerous efforts in this area are being made in many countries. Theoretically, if complete NOTES could be performed, an abdominal incision would be absent; hence, from the aspect of cosmetics or complications, results better than those from previous laparotomy or laparoscopic surgery could be obtained, and shorter time to return to work would be possible. Development of proper NOTES equipment will remove one of the present limitations of NOTES surgery do that it could be applied to more surgical fields.

Currently, several problems should be resolved for the further application of NOTES techniques to humans. First is the limitation of access routes. The ultimate goal of NOTES is to perform intraabdominal surgery without abdominal incision through the stomach, colon or bladder. However, proper closure methods for the routes of organs after intraabdominal manipulation are not available. In animal experiments, successful closure using endoscopic clips has been reported; nonetheless, experiences in humans are still not sufficient. Park et al. [7] attempted closure of the stomach wall using T-tags in humans. In their study, one case out of 2 developed severe complications, and they suggested that application of NOTES to humans still had many difficulties. Presently, pure NOTES using an approach through the stomach, colon and bladder is not possible, and hybrid methods using one or two abdominal incisions in combination are widely used. Second is an ethical problem. Because of the limitation of an intraabdominal approach, a transvaginal route, which is applicable to only women, is preferred. However, controversy still exists from an ethical aspect. Reviewing the result of a questionnaire survey conducted by Sung et al. on women receiving a cholecystectomy [8], the percentage of women wanting to undergo a transvaginal approach was $38 \%$, which is less than half. The results of a survey performed by Strickland et al. [9] on 300 women regarding the willingness to undergo the transvaginal approach NOTES show that approximately $70 \%$ of the women expressed a negative opinion. To resolve such ethical problems, the ethical committee, as well as the committee for clinical trials, of each hospital should consider NOTES carefully, and it should be performed after obtaining informed consents from the patient.

In regard to our surgery, the institutional review board of our hospital recommends that patients of child-bearing age be excluded due to risk of infertility and that a hybrid NOTES, rather than pure NOTES, be performed due to the limitations on the closure methods. In addition, the surgeon's experience is also important. The surgeon of this study performed more than 500 laparoscopic surgeries. including laparoscopic colectomies, and had experience with more than 100 cases of colonoscopy, and 3 cases of NOTES in animal experiments.

The sterilization of equipment is also an important factor. Generally, bacteriostatic sterilization is sufficient for routine endoscopic purposes; however, for intraperitoneal insertion, bacteriocidal sterilization is required. In our study, following the guideline suggested by the Minimal Access Therapy Decontamination Working Group, bacteriocidal sterilization was performed with ethylene oxide.

In contrast to pure NOTES, which is a technique using only endoscopy without abdominal incisions, hybrid NOTES refers to a procedure inserting one or two trocars in the abdominal area and using laparoscopic equipment. Presently, the safety of pure NOTES without the application of laparoscopic equipment has not been established yet; thus, hybrid methods are best for applications to humans. In a hybrid transvaginal endoscopic appendectomy, the first step is to prepare the pneumoperitoneum. In the pure NOTES procedure, the pneumoperitoneum is generated by a perforation of the vaginal posterior wall, and 
in the hybrid method, a 5-mm trocar is inserted in the vicinity of the umbilicus. Thus, if a trocar is used, the pneumoperitoneum and the perforation of the posterior vaginal wall can be performed safely. A limitation of the current endoscopic procedures is that the endoscopic equipment is not suitable for intraperitoneal surgeries. In regard to endoscopic forceps, the elasticity is high, and the hardness is low; thus, it is difficult to retract the appendix. In addition, concerning the endoscopic loop ligatures, the safety of the ligation of the base of appendix has not been established. Therefore, for time being, it is safe to use laparoscopic equipment such as laparoscopic loop ligatures. Recently in Europe, NOTES procedure equipment, such as the anubiscope, has been developed and is undergoing clinical trials [10]. In the future, if such equipment is introduced, the indications for NOTES should be safely and readily expanded.

\section{CONFLICT OF INTEREST}

No potential conflict of interest relevant to this article was reported.

\section{REFERENCES}

1. Kalloo AN, Singh VK, Jagannath SB, Niiyama H, Hill SL, Vaughn $\mathrm{CA}$, et al. Flexible transgastric peritoneoscopy: a novel approach to diagnostic and therapeutic interventions in the peritoneal cavity. Gastrointest Endosc 2004;60:114-7.

2. Marescaux J, Dallemagne B, Perretta S, Wattiez A, Mutter D, Coumaros D. Surgery without scars: report of transluminal cholecystectomy in a human being. Arch Surg 2007;142:823-6.

3. Rao GV, Reddy DN, Banerjee R. NOTES: human experience. Gastrointest Endosc Clin N Am 2008;18:361-70.
4. Shin EJ, Lee HJ, Chung JC, Chung GA, Chu CW, Cho GS, et al. Hybrid NOTES: transvaginal laparoscopic endoscopic appendectomy. In: The Semi-Annual Conference of the Korean Society of Endoscopic \& Laparoscopic Surgeons; 2007 Nov 11-9; Seoul. Seoul: The Korean Society of Endoscopic \& Laparoscopic Surgeons; 2007.

5. Kim YI, Park JH, Lee SI, Jeong SM, Kim JY. Transgastric gastroscopic intra-abdominal exploration in a female dog model: NOTES (natural orifice transluminal endoscopic surgery). J Korean Soc Coloproctol 2007;23:397-402.

6. First NOTES success in Korea. KorMedi news (2009.01.07) [Internet]. Seoul: KoreaMedicare Co., Ltd.; [cited 2010 Aug 20]. Available from: http://www.kormedi.com/news/article/1187570_2892. html.

7. Park PO, Bergstrom M. Transgastric peritoneoscopy and appendectomy: thoughts on our first experience in humans. Endoscopy 2010;42:81-4.

8. Sung JJ, Shin EJ, Song OP, Kim HC, Lim CW, Cho GS, et al. Patient perception of natural orifice transluminal endoxcopic surgery in cholecystectomy. In: The 26th Semi-Annual Conference of the Korean Society of Endoscopic \& Laparoscopic Surgeons; 2010 Jun 16-30; Seoul. Seoul: The Korean Society of Endoscopic \& Laparoscopic Surgeons; 2010.

9. Strickland AD, Norwood MG, Behnia-Willison F, Olakkengil SA, Hewett PJ. Transvaginal natural orifice translumenal endoscopic surgery (NOTES): a survey of women's views on a new technique. Surg Endosc 2010;24:2424-31.

10. Dallemagne B. An endoscopic platform: the ANUBISCOPE [Internet]. World Electronic Book of Surgery; [cited 2010 Aug 20]. Availabble from: http://www.websurg.com/ref/An_endoscopic_ platform\%253A_the_ANUBISCOPE-lt03endallemagne003.htm. 